\title{
Lipid Mediator Metabolomics via LC-MS/MS Profiling and Analysis
}

Jesmond Dalli ${ }^{1}$, Romain A. Colas ${ }^{1}$, Mary E. Walker ${ }^{1}$ and Charles N. Serhan ${ }^{2}$

${ }^{1}$ Lipid Mediator Unit, Biochemical Pharmacology, William Harvey Research Institute, Barts and the London School of Medicine, Queen Mary University of London, London,

United Kingdom. EC1M 6BQ

${ }^{2}$ Center for Experimental Therapeutics and Reperfusion Injury, Department of Anesthesiology, Perioperative and Pain Medicine, Brigham and Women's Hospital and Harvard Medical School, Boston, MA. USA 02115

Contact:

Dr Jesmond Dalli Ph.D, William Harvey Research Institute, John Vane Science Centre, Charterhouse Square, London. EC1M 6BQ. E-mail: j.dalli@qmul.ac.uk

Tel: +44 (0) 2078828263 


\begin{abstract}
Solid phase extraction coupled with liquid chromatography tandem mass spectrometry provides a robust and sensitive approach for the identification and quantitation of specialized pro-resolving mediators (lipoxins, resolvins, protectins and maresins), their pathway markers and the classic eicosanoids. Here, we provide a detailed description of the methodologies employed for the extraction of these mediators from biological systems, setup of the instrumentation, sample processing and then the procedures followed for their identification and quantitation.
\end{abstract}

\title{
Running header: Lipid Mediator Profiling
}

Key Words: Lipid mediator metabololipidomics, flux analysis, eicosanoids, resolvin, protectin, maresin, profiling, liquid chromatography-tandem mass spectrometry. 


\section{Introduction}

The role of lipid mediators in regulating distinct aspects of the body's functions in humans and experimental systems is well appreciated [1-4]. In this chapter, we shall detail the methodologies pioneered in the Serhan laboratory to obtain a snapshot of the dynamic pathways for the four major bioactive metabolomes that include the arachidonic acid eicosapentaenoic acid and docosahexaenoic acid metabolomes [5-12]. These methodologies provide insights into mechanisms activated during inflammation as well as new leads into underlying causes of disease by measuring the flux down each of the major bioactive metabolomes [5-11]. The methodologies that will be discussed herein employ C18 based extraction to enrich for lipid mediators in biological systems. These are then coupled with reverse phase liquid chromatography electrospray tandem mass spectrometry that allows for the separation, identification and quantitation of these molecules $[6,10]$. Given that the structures of these mediators are conserved throughout evolution, these methodologies are applicable to biological material from experimental systems such as tunicates [13], mice [5,7] and baboons [9] as well as humans $[6,8,14,15,11]$. Thus, they facilitate the direct translation of findings made in experimental systems to humans and vice versa.

\section{Materials}

All solvents should be LC/MS or analytical grade. All stocks should be handled using zero dead volume Hamilton syringes. Ensure that the Hamilton syringes are appropriately cleaned using isopropanol and methanol; this should be done by aliquoting at least 20 syringe volumes with each solvent. Please note that these compounds are sensitive to light, oxygen and heat. They should be stored at -20 ${ }^{\circ} \mathrm{C}$ or $-80{ }^{\circ} \mathrm{C}$, and exposure to light should be minimized. Each time the stock is used, purge the vial with a gentle stream of nitrogen very briefly before closing it to 
prevent oxidative degradation. DO NOT use these compounds with DMSO because the SPM are sensitive to isomerization and oxidation in this solvent.

\subsection{Sample preparation and Solid Phase Extraction}

1. Methanol $(\mathrm{MeOH})$ is used for protein precipitation and sample extraction as well as solid phase extraction (SPE) cartridge equilibration

2. For washing of SPE cartridges use $n$-hexane

3. Methyl formate is used as SPE elution solvent

4. Isolute $500 \mathrm{mg} / 3 \mathrm{~mL}$ C18 SPE columns are used(Biotage)

5 Deuterium-labelled internal standards used for quantification can be found inTable 1.

1.

2.

\subsection{Liquid chromatography tandem mass spectrometry}

1. Water containing $0.01 \%$ acetic acid is used as solvent $A$

2. $\mathrm{MeOH}$ containing $0.01 \%$ acetic acid is used as solvent $B$

3. LC-MS standards (see Table 2-4) 
4. A Poroshell $120 \mathrm{EC}-184.6 \mathrm{~mm} \times 100 \mathrm{~mm} \times 2.7 \mu \mathrm{m}$ reversed phase column is employed (Agilent)

\section{Methods}

\subsection{Preparing standard mixes for internal standards and standard curves}

\subsubsection{Determining the concentration of synthetic material}

1. Blank the UV spectrophotometer using the solvent of interest.

2. Aliquot a known volume of the stock using a Hamilton syringe

3. Measure the UV absorbance ensuring that a characteristic chromophore is observed for the molecule of interest (i.e. diene, triene or tetraene) [6]

4. Calculate absorption using a 3 point dropline

5. Calculate concentration using Beer-Lambert law:

Concentration $=$ absorbance/extinction coefficient ${ }^{*}$ dilution $($ See Table 5 for $\lambda_{\max }{ }^{\mathrm{MEOH}}$ and extinction co-efficients for each conjugated double bond system)

\subsection{Tuning of the LC-MS-MS for Lipid mediator profiling}

1. Using synthetic standards, first tune the probe position to maximize signal

2. Next, adjust electrode position

3. Afterwards, in the instrument parameters window adjust curtain gas, collisionally activated dissociation (CAD) gas, electrode voltage, source temperature and the source gases one at a time to identify optimal source settings.

4. Subsequently tune the compound parameters (Declustering Potential, Entrance Potential, Collision Energy, and Collision Cell Exit Potential) for each compound and for each transition

5. It is suggested that for each compound (both pathway markers \& mediators) at least 2 transitions are used 


\subsection{Automated Lipid Mediator extraction}

\subsubsection{Sample preparation}

1. Ensure that samples have not been freeze-thawed, fresh samples are preferred

2. For frozen samples allow to defrost on ice

3. For tissues dissociate gently in ice-cold $\mathrm{MeOH}$ ensuring that the samples remain cold throughout the process.

4. For liquid samples (e.g. cell culture preparations and plasma) add 4 equal volumes of ice-cold methanol containing deuterium-labelled internal standards to each sample

5. Place at $-20^{\circ} \mathrm{C}$ for $45 \mathrm{~min}$ to allow for protein precipitation

6. Centrifuge samples at $2000 \times \mathrm{g}$ for $10 \mathrm{~min}$ at $4{ }^{\circ} \mathrm{C}$

7. Collect supernatant

8. Transfer tubes to TurboVap Evaporator

9. Ensure that the water bath is set to $37^{\circ} \mathrm{C}$

10. Turn nitrogen feed on and set the flow rate to no more than $15 \mathrm{psi}$

11. Ensure that the lid is closed

12. Methanol volume should be evaporated to less than $1 \mathrm{~mL}$ using a steady nitrogen stream

13. Centrifuge samples at $2000 \times \mathrm{g}$ for $10 \mathrm{~min}$ at $4^{\circ} \mathrm{C}$

14. Samples are now ready for extraction. At this stage samples should not be stored

\subsubsection{Lipid Mediator Extraction}

1. Add new $\mathrm{C} 18$ columns on the rack

2. Add the collection plates containing elution tubes

3. Make sure run through plate is at position $D$

4. Click "run single method" 
5. Select the extraction method

6. Click "prepare run"

7. Go to solvent feeder (5) and prime each individually (make sure, the containers are filled, especially the MF)

8. Go to extraction media (3) and select how many columns you will use

9. Go to solvent tips (1) and sample tips (2) and check tips are filled as displayed on screen. If necessary, add or delete missing columns (or add tips)

10. Click "run method"

11. Monitor that the extraction procedure initiated correctly

12. At the end of the extraction, transfer the eluted samples (i.e. the MF fraction) to glass tubes, rinse the collection tube or the collecting well once with $\mathrm{MeOH}$ and add to the sample

13. Samples are ready for drying on Turbovap

\subsubsection{Solvent Evaporation}

1. Switch the TurboVap Evaporator on

2. Ensure that the water bath is set to $37^{\circ} \mathrm{C}$

3. Turn nitrogen feed on and set the flow rate to no more than $15 \mathrm{psi}$

4. Place $10 \mathrm{~mL}$ conical borosilicate tubes containing the methylformate fraction or methanol fraction obtained during solid phase extraction in the TurboVap

5. Ensure that the lid is closed

6. When the solvent is more than $95 \%$ evaporated rinse the walls of the tube using methylformate

7. When the solvent is more than $95 \%$ evaporated rinse the walls of the tube using methanol

8. When the solvent is completely evaporated, add $40 \mu \mathrm{l}$ of methanol/water (1:1)

9. Centrifuge the tube at $2000 \times \mathrm{g}$ for $2 \mathrm{~min}$

10. Carefully transfer the supernatant to an injection vial insert 
11. Place the insert in a clearly labelled $1.5 \mathrm{~mL}$ eppendorf tube

12. Centrifuge the insert at $10000 \times g$ for no more than $2 \mathrm{~min}$

13. Transfer the supernatant to an injection vial insert and place the insert in a clearly labelled sample vial.

14. Samples are now ready for LC-MS-MS profiling.

\subsection{Chromatography}

1. Using an Poroshell $120 \mathrm{EC}-18(4.6 \mathrm{~mm} \times 100 \mathrm{~mm} \times 2.7 \mu \mathrm{m})$ and water containing $0.01 \%$ acetic acid as solvent $A$, and methanol containing $0.01 \%$ acetic acid as solvent $B$, the following gradient should be used to chromatographically separate the mediators from their isomers and pathway markers

2. Column temperature should be set at $50{ }^{\circ} \mathrm{C}$

3. Equilibrate the column with mobile phase at $80: 20(A: B)$

4. This should be ramped to $50: 50(A: B)$ over 12 seconds

5. The gradient should be maintained for 2 minutes

6. Then to $80: 20$ over the subsequent 9 min

7. This should be maintained for the next $3.5 \mathrm{~min}$

8. Then ramped to $98: 2(A: B)$

9. Finally maintain this for 5.4 min to wash the column

10. The follow rate should be maintained at $0.5 \mathrm{~mL} / \mathrm{min}$ throughout the experiment

\subsection{Data analysis using Analyst Quantitate tool}

\subsubsection{Setting up a quantitation method}

1. In Analyst Click on Build Quantitation Method

2. Select a file that contains a standard mix

3. Assign which transitions correspond to the internal standards 
4. Assign the internal standards that will be used for identification and quantitation of each molecule.

5. In the Integrate tab select a retention time window of not more than 5 seconds

6. Save the method

\subsubsection{Analysing the data}

1. Click the quantitation wizard and follow the instructions to load your samples and the appropriate analysis method obtained as detailed above

2. Start by integrating the deuterium-labelled internal standards making a note of any drifts from the expected retention times

3. If retention-time drifts are observed correct the expected retention times for each of the analyte according to the retention time drift observed in the respective internal standards being used for identification (e.g. if $d_{5}-L X A_{4}$ is observed to elute 2 seconds after the anticipated retention time then $\mathrm{LXA}_{4}$ expected retention time will also shift by 2 seconds)

4. After determining the expected retention times for each of the samples integrate each of the mediators. Here all peaks that are clearly visible even if not more than 3 times higher than baseline should be integrated.

5. After integrating all the peaks in explore mode search for MS-MS spectra for molecules where a peak was obtained in at least 1 transition in the same region of the chromatogram where the peak was recorded.

6. Compare the tandem mass spectrum obtained in the sample with that obtained from the synthetic/authentic compound matching at least 6 ions in the MS-MS spectrum, with one of the ions being derived from a backbone break. These can be compared and matched to the Spectra Book available for all of these mediators and pathways on the Serhan lab website. 
7. For those molecules where the MS-MS spectrum is a positive match proceed to quantitation using a standard curve constructed using either synthetic or authentic standard mixes with concentrations determined as detailed above.

8. For molecules where standards are not available, molecules that carry similar physical properties may be used.

9. If an MS-MS spectrum is not obtained for a molecule or group of molecules and additional sample is available this could be re-run looking specifically for the molecule(s) where a spectrum was not obtained.

10. If no additional sample is available then the identification criteria are not fulfilled.

\section{Notes}

1. Concentrated $\mathrm{HCl}$ and acetic acid should be handled in a fumehood. Gloves and eye protection need to be wore at all times when handling these acids. Also always add the acid to water to avoid injury..

2. All standard stocks should be stored under nitrogen, shielded from light and at $20^{\circ} \mathrm{C}$ for short-term storage and $-80^{\circ} \mathrm{C}$ for long-term storage.

3. Each time the stock is used, purge the vial with a gentle stream of nitrogen very briefly before closing it to prevent oxidative degradation.

4. All solvents are flammable, they should be handled with care in a fumehood and kept away from open flames

5. All compound stocks should be handled using zero dead volume Hamilton syringes

6 Ensure that the Hamilton syringes are appropriately cleaned using isopropanol and methanol; this should be done by aliquoting at least 20 syringe volumes with each solvent. 
7. Only use nitrogen or an inert gas to evaporate solvents, do not use air since this will lead to mediator oxidation.

8 Upon solvent evaporation and suspension of samples in water/methanol samples need to be profiled within $24 \mathrm{~h}$ to avoid isomerization of the mediators. At all times samples should be kept at $4^{\circ} \mathrm{C}$ and not frozen to maximise mediator integrity.

9. DO NOT DMSO at any stage since SPM are sensitive to isomerization and oxidation in this solvent.

\section{Acknowledgements}

This work was supported by funding from the European Research Council (ERC) under the European Union's Horizon 2020 research and innovation programme (Grant number: 677542), a Sir Henry Dale Fellowship jointly funded by the Wellcome Trust and the Royal Society (Grant number: 107613/Z/15/Z) and the Barts Charity (Grant number:

MGU0343). CN Serhan is supported by the National Institutes of Health, USA Grant Number P01GM095467. 


\section{$\underline{\text { References }}$}

1. Basil MC, Levy BD (2016) Specialized pro-resolving mediators: endogenous regulators of infection and inflammation. Nat Rev Immunol 16 (1):51-67. doi:10.1038/nri.2015.4 2. Haworth O, Buckley CD (2015) Pathways involved in the resolution of inflammatory joint disease. Semin Immunol 27 (3):194-199. doi:10.1016/j.smim.2015.04.002 3. Miyata J, Arita M (2015) Role of omega-3 fatty acids and their metabolites in asthma and allergic diseases. Allergol Int 64 (1):27-34. doi:10.1016/j.alit.2014.08.003 4. Serhan CN, Chiang N, Dalli J (2015) The resolution code of acute inflammation: Novel pro-resolving lipid mediators in resolution. Semin Immunol 27 (3):200-215. doi:10.1016/j.smim.2015.03.004

5. Chiang N, Fredman G, Backhed F, Oh SF, Vickery T, Schmidt BA, Serhan CN (2012) Infection regulates pro-resolving mediators that lower antibiotic requirements. Nature 484 (7395):524-528. doi:10.1038/nature11042

6. Colas RA, Shinohara M, Dalli J, Chiang N, Serhan CN (2014) Identification and signature profiles for pro-resolving and inflammatory lipid mediators in human tissue. Am J Physiol Cell Physiol 307 (1):C39-54. doi:10.1152/ajpcell.00024.2014

7. Dalli J, Colas RA, Arnardottir H, Serhan CN (2017) Vagal Regulation of Group 3 Innate Lymphoid Cells and the Immunoresolvent PCTR1 Controls Infection Resolution. Immunity 46 (1):92-105. doi:10.1016/j.immuni.2016.12.009

8. Dalli J, Colas RA, Quintana C, Barragan-Bradford D, Hurwitz S, Levy BD, Choi AM, Serhan CN, Baron RM (2017) Human Sepsis Eicosanoid and Proresolving Lipid Mediator Temporal Profiles: Correlations With Survival and Clinical Outcomes. Crit Care Med 45 (1):58-68. doi:10.1097/CCM.0000000000002014

9. Dalli J, Kraft BD, Colas RA, Shinohara M, Fredenburgh LE, Hess DR, Chiang N, WeltyWolf K, Choi AM, Piantadosi CA, Serhan CN (2015) The Regulation of Proresolving Lipid 
Mediator Profiles in Baboon Pneumonia by Inhaled Carbon Monoxide. Am J Respir Cell Mol Biol 53 (3):314-325. doi:10.1165/rcmb.2014-02990C

10. Dalli J, Serhan CN (2012) Specific lipid mediator signatures of human phagocytes: microparticles stimulate macrophage efferocytosis and pro-resolving mediators. Blood 120 (15):e60-72. doi:10.1182/blood-2012-04-423525

11. Weiss GA, Troxler H, Klinke G, Rogler D, Braegger C, Hersberger M (2013) High levels of anti-inflammatory and pro-resolving lipid mediators lipoxins and resolvins and declining docosahexaenoic acid levels in human milk during the first month of lactation. Lipids Health Dis 12:89. doi:10.1186/1476-511X-12-89

12. Serhan CN, Petasis NA (2011) Resolvins and protectins in inflammation resolution. Chem Rev 111 (10):5922-5943. doi:10.1021/cr100396c

13. Knight J, Taylor GW, Wright P, Clare AS, Rowley AF (1999) Eicosanoid biosynthesis in an advanced deuterostomate invertebrate, the sea squirt (Ciona intestinalis). Biochim Biophys Acta 1436 (3):467-478

14. Rathod KS, Kapil V, Velmurugan S, Khambata RS, Siddique U, Khan S, Van Eijl S, Gee LC, Bansal J, Pitrola K, Shaw C, D'Acquisto F, Colas RA, Marelli-Berg F, Dalli J, Ahluwalia A (2017) Accelerated resolution of inflammation underlies sex differences in inflammatory responses in humans. J Clin Invest 127 (1):169-182. doi:10.1172/JCl89429 15. Titos E, Rius B, Lopez-Vicario C, Alcaraz-Quiles J, Garcia-Alonso V, Lopategi A, Dalli J, Lozano JJ, Arroyo V, Delgado S, Serhan CN, Claria J (2016) Signaling and Immunoresolving Actions of Resolvin D1 in Inflamed Human Visceral Adipose Tissue. J Immunol 197 (8):3360-3370. doi:10.4049/jimmunol.1502522

\section{Tables}


Table 1 List of deuterated internal standards, their complete stereochemistries and source.

\begin{tabular}{|c|c|c|c|}
\hline Abbreviation & $\underline{\text { Trivial name }}$ & Full stereochemistry & Source \\
\hline $5 \mathrm{~S}-\mathrm{HETE}-\mathrm{d}_{8}$ & $\begin{array}{l}\text { Deuterium-labelled } \\
\text { 5S-HETE }\end{array}$ & $\begin{array}{l}\text { 5S-hydroxy-eicosa-6E, 8Z,11Z,14Z-tetraenoic-5,6,8,9,11,12,14,15- } \\
d_{8} \text { acid }\end{array}$ & Cayman Chemicals \\
\hline $\mathrm{LTB}_{4}-\mathrm{d}_{4}$ & $\begin{array}{l}\text { Deuterium-labelled } \\
\text { leukotriene } \mathrm{B}_{4}\end{array}$ & $\begin{array}{l}\text { 5S,12R-dihydroxy-eicosa-6Z,8E,10E,14Z-tetraenoic-6,7,14,15- } \\
\mathrm{d}_{4} \text { acid }\end{array}$ & Cayman Chemicals \\
\hline$L X A_{4}-d_{5}$ & $\begin{array}{l}\text { Deuterium-labelled } \\
\text { lipoxin } A_{4}\end{array}$ & $\begin{array}{l}\text { 5S,6R,15S-trihydroxy-eicosa-7E,9E,11Z,13E-tetraenoic- } \\
\text { 19,19,20,20,20-d5 acid }\end{array}$ & Cayman Chemicals \\
\hline RvD2-d 5 & $\begin{array}{l}\text { Deuterium-labelled } \\
\text { resolvin D2 }\end{array}$ & $\begin{array}{l}\text { 7S,16R,17S-trihydroxy-docosa-4Z,8E,10Z,12E,14E,19Z-hexaenoic- } \\
\text { 21,21,22,22,22-d5 acid }\end{array}$ & Cayman Chemicals \\
\hline $\mathrm{PGE}_{2}-\mathrm{d}_{4}$ & $\begin{array}{l}\text { Deuterium-labelled } \\
\text { prostaglandin } E_{2}\end{array}$ & 9-oxo-11a,15S-dihydroxy-prosta-5Z,13E-dien-1-oic-3,3,4,4-d 4 acid & Cayman Chemicals \\
\hline
\end{tabular}

Table 2 List of arachidonic acid metabolome, their complete stereochemistries and source.

\begin{tabular}{|c|c|c|c|}
\hline Abbreviation & $\underline{\text { Trivial name }}$ & Full stereochemistry & $\underline{\text { Source }}$ \\
\hline AA & Arachidonic acid & eicosa-5Z,8Z,11Z,14Z-tetraenoic acid & Cayman Chemicals \\
\hline $\mathrm{PGD}_{2}$ & Prostaglandin $\mathrm{D}_{2}$ & 9a,15S-dihydroxy-11-oxo-prosta-5Z,13E-dien-1-oic acid & Cayman Chemicals \\
\hline $\mathrm{PGE}_{2}$ & Prostaglandin $\mathrm{E}_{2}$ & 9-oxo-11a,15S-dihydroxy-prosta-5Z,13E-dien-1-oic acid & Cayman Chemicals \\
\hline $\mathrm{PGF}_{2 a}$ & Prostaglandin $\mathrm{F}_{2 a}$ & 9a,11a,15S-trihydroxy-prosta-5Z,13E-dien-1-oic acid & Cayman Chemicals \\
\hline $\mathrm{TxB}_{2}$ & Thromboxane $\mathrm{B}_{2}$ & $9 \alpha, 11,15$ S-trihydroxy-thromba-5Z,13E-dien-1-oic acid & Cayman Chemicals \\
\hline $\mathrm{LTB}_{4}$ & Leukotriene $\mathrm{B}_{4}$ & 5S,12R-dihydroxy-eicosa-6Z,8E,10E,14Z-tetraenoic acid & Cayman Chemicals \\
\hline $\mathrm{LXA}_{4}$ & Lipoxin $\mathrm{A}_{4}$ & 5S,6R,15S-trihydroxy-eicosa-7E,9E,11Z,13E-tetraenoic acid & Cayman Chemicals \\
\hline $\mathrm{LXB}_{4}$ & Lipoxin $\mathrm{B}_{4}$ & 5S,14R,15S-trihydroxy-eicosa-6E,8Z,10E,12E-tetraenoic acid & Cayman Chemicals \\
\hline 15-HETE & & 15S-hydroxy-eicosa-5Z,8Z,11Z,13E-tetraenoic acid & Cayman Chemicals \\
\hline 12-HETE & & 12-hydroxy-eicosa-5Z,8Z,10E,14Z-tetraenoic acid & Cayman Chemicals \\
\hline 5-HETE & & 5S-hydroxy-eicosa-6E,8Z,11Z,14Z-tetraenoic acid & Cayman Chemicals \\
\hline$\Delta 6$-trans-LTB 4 & $\begin{array}{l}\Delta 6 \text {-trans- } \\
\text { leukotriene } B_{4}\end{array}$ & $5 \mathrm{~S}, 12 \mathrm{R}$-dihydroxy-eicosa-6E,8E,10E,14Z-tetraenoic acid & Cayman Chemicals \\
\hline 5S,12S-diHETE & & 5S,12S-dihydroxy-eicosa-6E,8E,10E,14Z-tetraenoic acid & Biogenic synthesis \\
\hline
\end{tabular}


Table 3 List of eicosapentaenoic acid metabolome, their complete stereochemistries and source.

\begin{tabular}{|c|c|c|c|}
\hline Abbreviation & Trivial name & Full stereochemistry & \\
\hline EPA & & eicosa-5Z,8Z,11Z,14Z,17Z-pentaenoic acid & Cayman Chemicals \\
\hline RvE1 & Resolvin E1 & $\begin{array}{l}5 S, 12 R, 18 R \text {-trihydroxy-eicosa-6Z,8E,10E,14Z,16E-pentaenoic } \\
\text { acid }\end{array}$ & Cayman Chemicals \\
\hline RvE2 & Resolvin E2 & 5S,18R-dihydroxy-eicosa-6Z,8E,11E,14E,16Z-pentaenoic acid & Biogenic synthesis \\
\hline RvE3 & Resolvin E3 & $\begin{array}{l}17 R, 18 R / S \text {-dihydroxy-eicosa-5Z,8Z,11Z,13E,15E-pentaenoic } \\
\text { acid }\end{array}$ & $\begin{array}{l}\text { Custom Synthesis (Dr } \\
\text { Makoto Arita, Riken } \\
\text { Institute Japan) }\end{array}$ \\
\hline 18-HEPE & & 18-hydroxy-eicosa-5Z,8Z,11Z,14Z,16E-pentaenoic acid & Cayman Chemicals \\
\hline 15-HEPE & & 15-hydroxy-eicosa-5Z,8Z,11Z,13E,17Z-pentaenoic acid & Cayman Chemicals \\
\hline 12-HEPE & & 12-hydroxy-eicosa-5Z,8Z,10E,14Z,17Z-pentaenoic acid & Cayman Chemicals \\
\hline 5-HEPE & & 5-hydroxy-eicosa-6E,8Z,11Z,14Z,17Z-pentaenoic acid & Cayman Chemicals \\
\hline
\end{tabular}


Table 4 List of docosahexaenoic acid metabolome, their complete stereochemistries and source.

\begin{tabular}{|c|c|c|c|}
\hline Abbreviation & Trivial name & Full stereochemistry & \\
\hline DHA & & docosa-4Z,7Z,10Z,13Z,16Z,19Z-hexaenoic acid & Cayman Chemicals \\
\hline RvD1 & Resolvin D1 & $\begin{array}{l}7 S, 8 R, 17 S \text {-trihydroxy-docosa-4Z,9E,11E,13Z,15E,19Z-hexaenoic } \\
\text { acid }\end{array}$ & Cayman Chemicals \\
\hline RvD2 & Resolvin D2 & $\begin{array}{l}\text { 7S,16R,17S-trihydroxy-docosa-4Z,8E, 10Z,12E,14E,19Z-hexaenoic } \\
\text { acid }\end{array}$ & Cayman Chemicals \\
\hline RvD3 & Resolvin D3 & $\begin{array}{l}\text { 4S,7R,17S-trihydroxy-docosa-5Z,7E,9E,13Z,15E,19Z-hexaenoic } \\
\text { acid }\end{array}$ & Cayman Chemicals \\
\hline RvD4 & Resolvin D4 & $\begin{array}{l}\text { 4S,5R,17S-trihydroxy-docosa-6E,8E, 10Z,13Z,15E,19Z-hexaenoic } \\
\text { acid }\end{array}$ & $\begin{array}{l}\text { Custom Synthesis (Dr } \\
\text { Charles Serhan }\end{array}$ \\
\hline RvD5 & Resolvin D5 & 7S,17S-dihydroxy-docosa-4Z,8E,10Z,13Z,15E,19Z-hexaenoic acid & Cayman Chemicals \\
\hline RvD6 & Resolvin D6 & 4S,17S-dihydroxy-docosa-5E,7Z,10Z,13Z,15E,19Z-hexaenoic acid & Biogenic Synthesis \\
\hline MaR1 & Maresin 1 & $7 R, 14 S$-dihydroxy-docosa-4Z,8E,10E,12Z,16Z,19Z-hexaenoic acid & Cayman Chemicals \\
\hline 4S,14S-diHDHA & & 4S,14S-dihydroxy-docosa-5Z,7E,10E,12Z,16E,19E-hexaenoic acid & Biogenic Synthesis \\
\hline 7S,14S-diHDHA & & 7S,14S-dihydroxy-docosa-4Z,8E,10E,12Z,16E,19E-hexaenoic acid & Biogenic Synthesis \\
\hline PD1 & Protectin D1 & 10R,17S-dihydroxy-docosa-4Z,7Z,11E,13E,15Z,19Z-hexaenoic acid & $\begin{array}{l}\text { Custom Synthesis (Dr } \\
\text { Charles Serhan) }\end{array}$ \\
\hline 10S,17S-diHDHA & Protectin Dx & 10S,17S-dihydroxy-docosa-4Z,7Z,11E,13Z,15E,19Z-hexaenoic acid & Cayman Chemicals \\
\hline 22-OH-PD1 & $\begin{array}{l}\text { 22-OH- } \\
\text { Protectin D1 }\end{array}$ & $\begin{array}{l}\text { 10R,17S,20-trihydroxy-docosa-4Z,7Z,11E,13E,15Z,19Z-hexaenoic } \\
\text { acid }\end{array}$ & $\begin{array}{l}\text { Custom Synthesis (Dr } \\
\text { Trond V. Hansen, } \\
\text { University of Oslo) }\end{array}$ \\
\hline 17-HDHA & & 17-hydroxy-docosa-4Z,7Z,10Z,13Z,15E,19Z-hexaenoic acid & Cayman Chemicals \\
\hline 14-HDHA & & 14S-hydroxy-docosa-4Z,7Z,10Z,12E, 16Z,19Z-hexaenoic acid & Cayman Chemicals \\
\hline 13-HDHA & & 13-hydroxy-docosa-4Z,7Z,10Z,14E, 16Z,19Z-hexaenoic acid & Cayman Chemicals \\
\hline 7-HDHA & & 7-hydroxy-docosa-4Z,8E, 10Z,13Z,16Z,19Z-hexaenoic acid & Cayman Chemicals \\
\hline 4-HDHA & & 4-hydroxy-docosa-5E,7Z,10Z,13Z,16Z,19Z-hexaenoic acid & Cayman Chemicals \\
\hline
\end{tabular}


Table 4 List of aspirin triggered mediators, their complete stereochemistries and source.

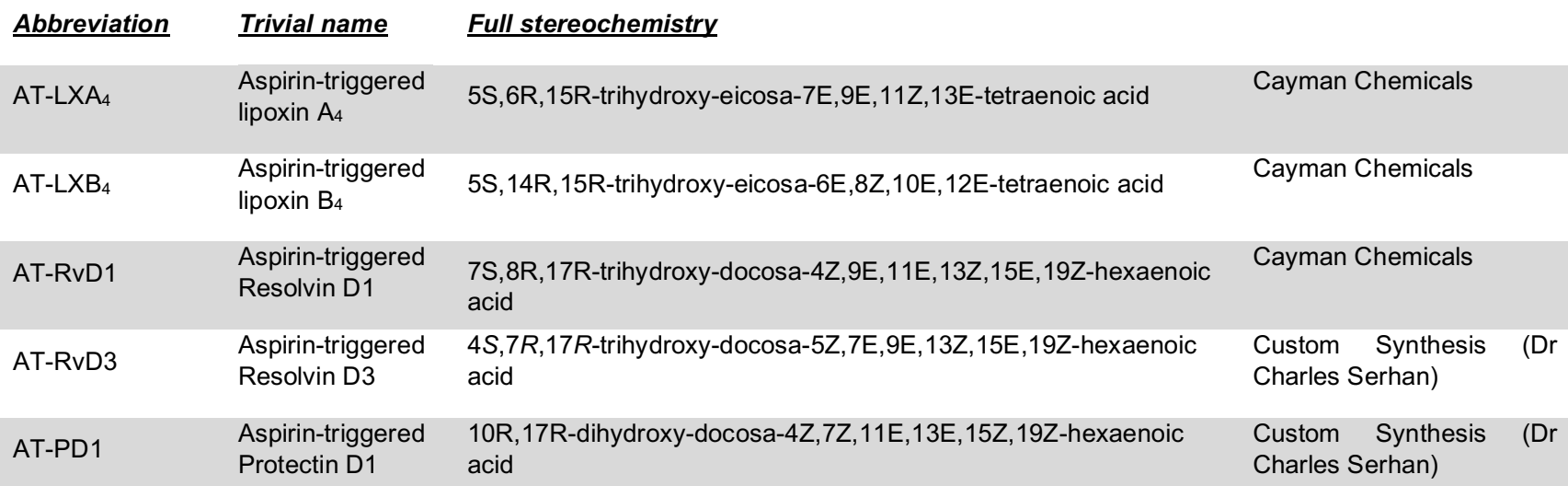

Table 5 Extinction co-efficient and $\lambda_{\max }{ }^{\mathrm{MEOH}}$ for distinct conjugated double bond systems

\begin{tabular}{|l|l|l|}
\hline Double bond system & Extinction Co-efficient & $\lambda_{\max }{ }^{\mathrm{MEOH}}$ \\
\hline $\begin{array}{l}\text { Conjugated diene } \\
\text { (e.g:monohydroxy acids) }\end{array}$ & 25,000 & 235 \\
\hline $\begin{array}{l}\text { Two conjugated dienes (e.g. } \\
\text { RvD5) }\end{array}$ & 25,000 & 240 \\
\hline $\begin{array}{l}\text { Diene-triene conjugated } \\
\text { system (e.g. RvE1) }\end{array}$ & 40,000 & 271 \\
\hline $\begin{array}{l}\text { Conjugated Triene (e.g. PD1) } \\
\text { Conjugated Tetraene (e.g. } \\
\text { RvD1) }\end{array}$ & 50,000 & 269 \\
\hline
\end{tabular}




\section{Figure Legends}

Figure 1. Sample preparation. Tissues from various origins (human or animal tissues or fluids, mice, planaria, cell culture, etc.) should be homogenized in ice-cold $\mathrm{MeOH}$ containing deuterated internal standards. Samples should always be kept on ice to prevent mediator isomerization. For biological fluids 4 volumes of methanol should be added to the samples. Homogenized tissues and biological fluids should then be kept at $20^{\circ} \mathrm{C}$ for $45 \mathrm{~min}$ to allow for protein precipitation. Samples should then be centrifuged, supernatant collected and acidified to $\mathrm{pH} 3.5$ with $\mathrm{HCl}$ prior to solid phase extraction. The eluting fraction containing the bioactive lipid mediators is dried under a gentle flux of nitrogen $\left(\mathrm{N}_{2}\right)$ and resuspended in phase prior injection. $\mathrm{MeOH}$, methanol; $\mathrm{H}_{2} \mathrm{O}$, water; $M F$, methyl formate; $\mathrm{N}_{2}$, nitrogen.

Figure 2. Schematic of instrument setup for mediator identification. Samples in phase are injected using a HPLC-MS-MS system, no more than $40 \mu$ l should be injected. LC, liquid chromatography; RP, reverse phase; $\mathrm{MeOH}$, methanol; ESI, electron spay ionization; $\mathrm{m} / \mathrm{z}$, mass to charge ratio; MRM, multiple reaction monitoring; EPI, enhanced product ion

Figure 3. Lipid mediator identification and quantification. Identification of bioactive LM is performed by matching retention time (RT) and at least 6 diagnostic ions from the MS-MS spectrum with those of synthetic or authentic standards for each mediator. Quantitation is achieved using linear regression curves that are constructed using synthetic or authentic standards for each mediator of interest. Interrelationship(s) for identified mediators within each condition and between different conditions is further 
investigated using multivariate analysis. LM, lipid mediator; RT, retention time; AUC, area under the curve; PCA, principal component analysis; PLS-DA, partial least squarediscriminant analysis; OPLS-DA, orthogonal partial least square-discriminant analysis 